\title{
Clinical outcomes of transoral robotic-assisted surgery for the management of head and neck cancer
}

\author{
Anuraag Parikh ${ }^{1, *}$ \\ Derrick Lin ${ }^{1,2}$ \\ Neerav Goyal ${ }^{3, *}$ \\ 'Department of Otolaryngology, \\ Massachusetts Eye and Ear Infirmary, \\ ${ }^{2}$ Department of Otology and \\ Laryngology, Harvard Medical \\ School, Boston, MA, ${ }^{3}$ Division of \\ Otolaryngology Head and Neck \\ Surgery, Penn State Milton S Hershey \\ Medical Center, Hershey, PA, USA \\ *These authors contributed equally to \\ this work
}

Correspondence: Neerav Goyal Division of Otolaryngology Head and Neck Surgery, Penn State Milton S. Hershey Medical Center, 500 University Drive, MC H09I, Hershey, PA I7033, USA

Tel +l 71753 I 8945

Email ngoyal !@hmc.psu.edu
This article was published in the following Dove Press journal:

Robotic Surgery: Research and Reviews

30 September 2015

Number of times this article has been viewed

Background: Transoral robotic surgery (TORS) has existed for nearly a decade as a treatment option for cancers of the upper aerodigestive tract. A number of Phase II clinical trials, mostly single-arm, are under way systematically examining outcomes of TORS, but the majority of the current literature is retrospective in nature.

Objective: The objective of this work was to review the functional and oncologic outcomes of TORS in the treatment of head and neck squamous cell carcinoma broken down by subsite.

Methods: A comprehensive search was performed using the MEDLINE database and keywords ("transoral robotic surgery" OR “TORS"). Articles specifically related to our objectives were included in our review.

Results: Twenty-six studies describing the functional and oncologic outcomes of TORS were reviewed, with 15 focusing on oropharyngeal cancers and the remainder on laryngeal and unknown primary lesions. TORS was associated with decreased need for free flap reconstruction, hospital stay, postoperative complications, and tracheostomy or gastrostomy tube dependence than open surgery, with at least comparable oncologic outcomes. In comparison with chemoradiation, TORS oropharyngectomy had similar subjective swallowing outcomes, comparable oncologic outcomes, and decreased gastrostomy tube dependence. Patient reported speech and overall health outcomes following TORS oropharyngectomy were equivocal. For patients with unknown primary lesions, the addition of TORS lingual and palatine tonsillectomy significantly increased the rate of identification of the primary site to greater than $70 \%$.

Conclusion: Although TORS is a promising treatment option for head and neck squamous cell carcinoma, further prospective trials are needed to more systematically assess outcomes and compare them with other treatment modalities, particularly chemoradiation therapy.

Keywords: TORS, oropharynx cancer, larynx cancer, unknown primary, outcomes

\section{Introduction}

The safety of transoral robotic surgery (TORS) was established nearly a decade ago, ${ }^{1}$ and TORS was first approved by the Food and Drug Administration in 2009 for benign and malignant tumors classified as T1 and T2. ${ }^{2}$ Approval for benign base of tongue procedures was added in $2014,{ }^{2}$ but there has been no further expansion in approved indications for the treatment of malignancy. With the advent of intensity modulated radiation (IMRT), which limits the toxicities traditionally associated with three-dimensional conformal radiation therapy (RT) and affords similar disease control, ${ }^{1}$ there has been interest in systematically investigating functional and oncologic outcomes of TORS. A number of single-arm Phase II clinical trials investigating these issues are under way. ${ }^{3}$ In addition, Phase II of the ORATOR trial at Case Western Reserve University 
is under way comparing RT with or without chemotherapy with TORS and selective neck dissection with or without adjuvant chemoradiation, with the primary end point being MD Anderson Dysphagia Index (MDADI) quality of life score and secondary end points of survival, toxicity, other quality of life outcomes, and swallowing function. ${ }^{4}$ Here, we aim to summarize the existing literature on functional and oncologic outcomes of TORS for the treatment of head and neck squamous cell carcinoma (HNSCC), divided by subsite, particularly in comparison with other available treatment modalities where possible.

\section{Methods}

A comprehensive review of the literature was performed using the MEDLINE database as indexed by PubMed. The MEDLINE database was searched using the terms "transoral robotic surgery" OR "TORS." A total of 390 entries were screened by title and abstract. Articles were excluded if they were deemed to be unrelated to TORS (119) or HNSCC (66). Additional articles were excluded if the article did not specifically address patient outcomes (133). Finally, all non-English language publications were excluded (5). The full text of the remaining 67 articles was reviewed in further detail. Of these, 41 studies were eliminated due to inadequate stratification of patients by HNSCC subsite, sample sizes that were too small (eg, case reports), and duplicate series of patients, leaving 26 articles to be reviewed, including 15 oropharynx, nine larynx, and two unknown primary (Figure 1). Other relevant articles encountered, including one recent review on TORS, were used for additional background information, but data were not included in the tables detailing outcomes. This review was exempt from Institutional Board Review.

\section{Oropharynx \\ Patient sample}

Fifteen original studies (14 single institution and one multiinstitutional) reported on perioperative issues (Table 1) and oncologic outcomes (Table 2) of 585 patients with oropharyngeal squamous cell carcinoma (OPSCC) who underwent TORS as part of their treatment, either in a primary (494 patients, $84 \%$ ) or in a salvage (91 patients, 16\%) treatment setting. Of those whose neck management was reported, 524 patients $(95 \%)$ underwent unilateral or bilateral neck dissection, either simultaneously with the TORS resection of the primary tumor or in staged fashion. Of the 494 patients whose adjuvant treatment status was reported, radiation therapy was given to 157 patients (32\%), and concurrent chemoradiation therapy was given to 177 patients $(36 \%)$.

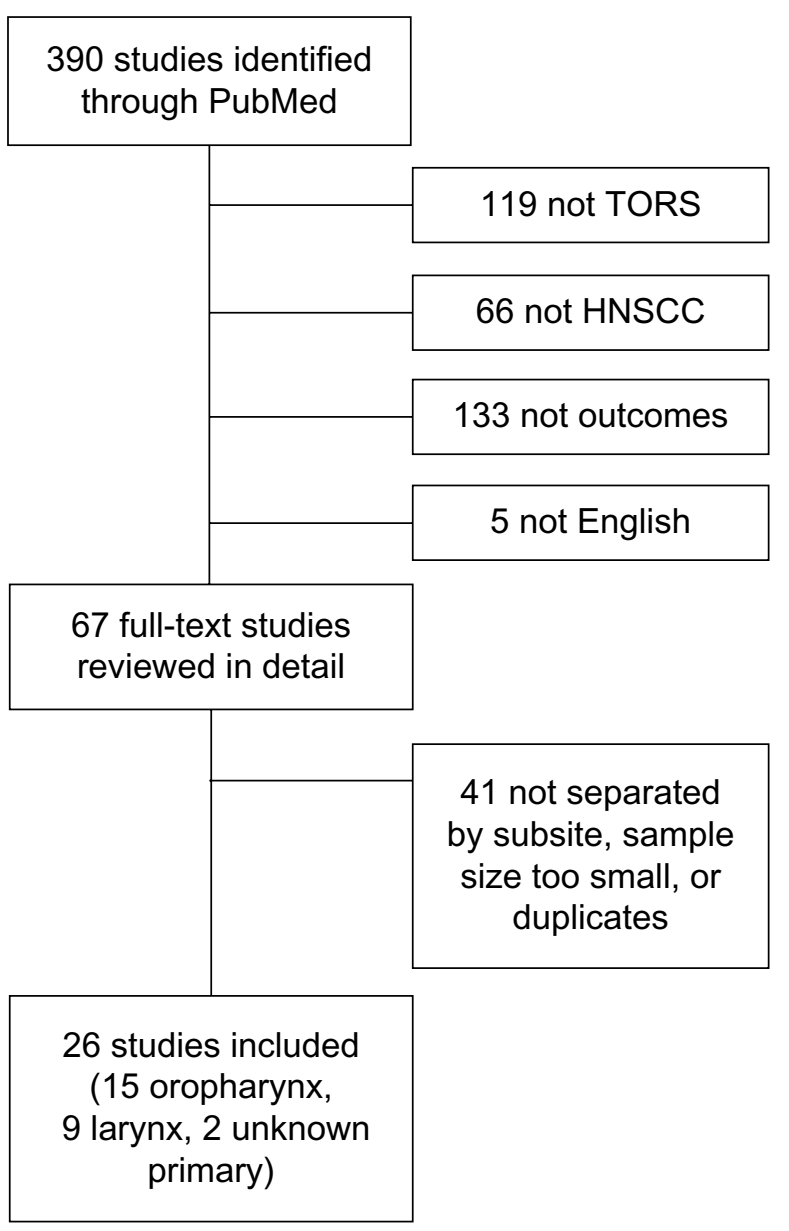

Figure I Flow diagram of included studies.

Abbreviations: HNSCC, head and neck squamous cell carcinoma; TORS, transoral robotic surgery.

A total of 126 patients (32\%) received no adjuvant therapy following surgery, secondary to either the lack of adverse pathologic features or refusal of adjuvant therapy.

\section{Need for free flap reconstruction}

Of nine studies reporting on the need for free flap reconstruction following TORS for OPSCC, five studies reported that no free flaps were used in any of their patients. In these case series, resection beds were allowed to granulate and heal by secondary intention..$^{5-9}$ Genden et $\mathrm{al}^{10}$ reported a series of 31 patients with OPSCC who underwent TORS and described the use of a musculomucosal advancement flap pharyngoplasty in 25 of these patients, with radial forearm free flap in the remaining six patients. However, they stated that four of these six patients were salvage candidates. Al-Khudari et $\mathrm{al}^{11}$ similarly found that of 22 patients undergoing TORS for OPSCC, nine required free flap reconstruction, and most of these patients were salvage candidates. These findings suggest a potentially increased need for free flap reconstruction 


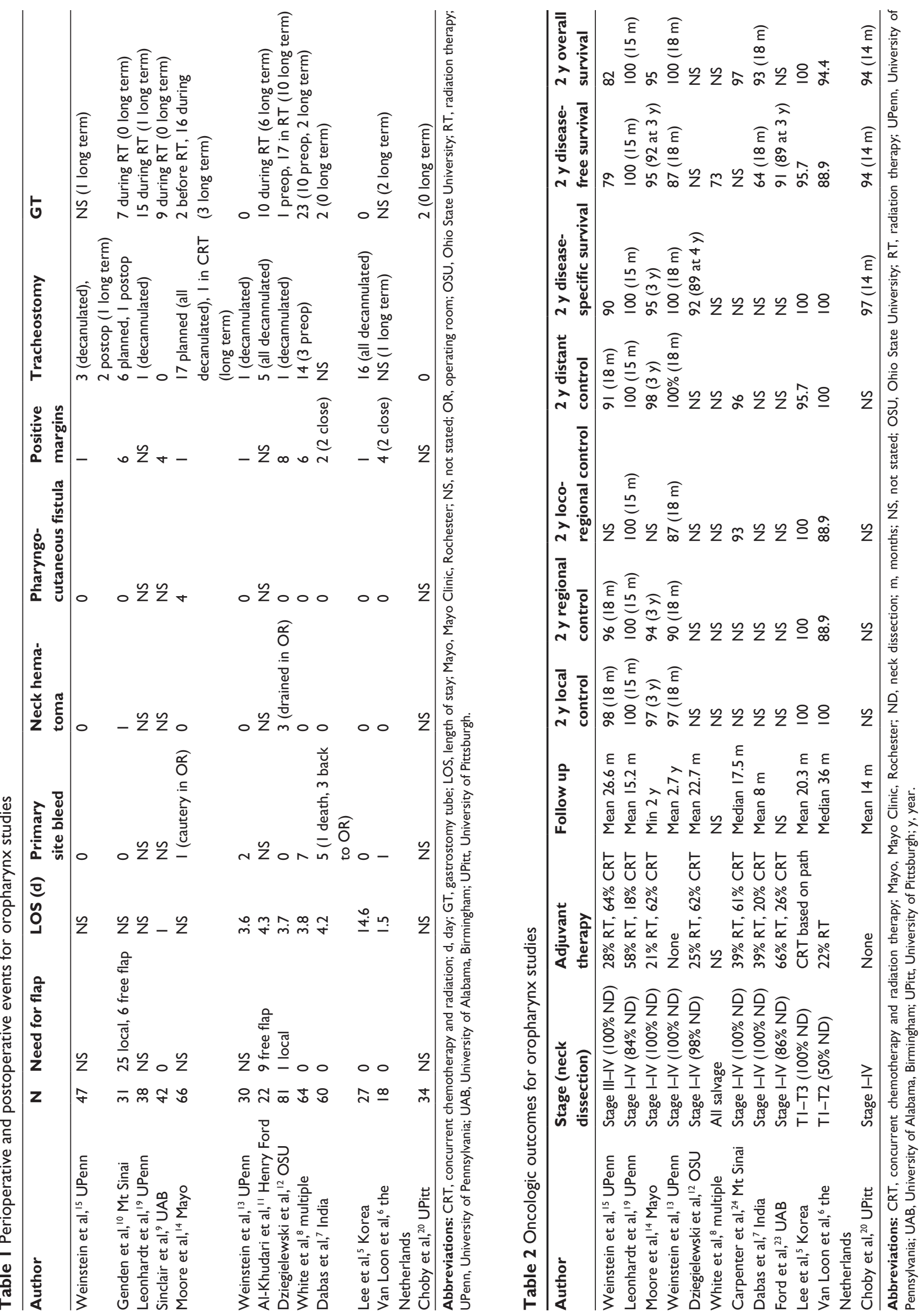


in patients undergoing salvage surgery. On the other hand, White et $\mathrm{al}^{8}$ reported a series of 64 patients with OPSCC undergoing salvage resection by TORS and stated that no free flaps were used for reconstruction.

Two studies investigated the need for free flap reconstruction following TORS in comparison with conventional open surgery for similar lesions. ${ }^{5,8}$ These studies reported that there was no need for free flap reconstruction in a series of 27 and 64 patients undergoing TORS for OPSCC. This was significantly less than the need for free flaps in 14/14 and 48/64 patients undergoing open surgery, respectively, ${ }^{5,8}$ strongly suggesting that although TORS patients may occasionally still require free flap reconstruction, particularly in the salvage setting, there is certainly a decreased need for free flaps as compared with conventional open surgery.

\section{Hospital length of stay}

The postoperative hospital length of stay (LOS) after TORS with or without neck dissection for OPSCC ranged from 1.0 days to 14.6 days, with all but one study reporting LOS in the range of 1.0-4.3 days. ${ }^{5-9,11-13}$ Studies comparing LOS following TORS with that following mandibulotomy for similar oropharyngeal lesions consistently report significantly shorter LOS with TORS, whether in the primary or salvage setting. ${ }^{5,8}$ Some part of this decreased LOS may be related to decreased need for free flap reconstruction, which typically necessitates intensive postoperative monitoring for longer periods of time, while part of it may be related to decreased time to oral intake, as discussed in subsequent sections.

\section{Surgical complications}

Minor postoperative bleeding from the primary resection site was reported in $0 \%-11 \%$ of patients, all within the first week after surgery. ${ }^{5-8,10,12-15}$ Two series reported of more serious postoperative bleeds: Dabas et $\mathrm{al}^{7}$ report on one patient who had bleeding and died, while van Loon et $\mathrm{al}^{6}$ report on one arterial hemorrhage that required tracheotomy and operative control of hemorrhage. Neck hematomas occurred in a similarly low fraction of patients, with published series reporting $0 \%-4 \%$ of cases with neck hematomas requiring operative drainage. ${ }^{5-8,10,12-15}$

Pharyngocutaneous fistula has been another common concern after TORS for OPSCC. Moore et $\mathrm{al}^{14}$ report four cases of pharyngocutaneous fistula out of 66 patients, all of which resolved with packing and required no operative intervention. Overall, published rates of pharyngocutaneous fistula were $0 \%-6 \%$, and all cases were managed nonoperatively. ${ }^{5-8,10,12-15}$
Despite the relatively low rates of fistula formation and straightforward management, many centers have adopted a strategy of staged neck dissection within 2-4 weeks after TORS to minimize risk. Moore et $\mathrm{al}^{16}$ assessed the impact of concurrent neck dissection on rates of fistula formation. Of 148 patients who underwent TORS with concomitant neck dissection, 42 were noted to have an orocervical communication intraoperatively. The majority $(90 \%)$ of these patients had tonsillar primary lesions, with only $10 \%$ having tongue base lesions. Small defects were managed by primary closure of pharyngeal constrictors, and defects larger than $1 \mathrm{~cm}$ were closed when possible and reinforced with muscular coverage. Six total fistulas ( $4 \%$ of all patients) were reported, all within 7-12 days after surgery. These were all controlled with conservative management, including NPO status, controlled bedside debridement, and packing with iodine impregnated gauze at the inferior aspect of the incision. There was no delay in adjuvant therapy and no need for operative intervention.

On the other hand, studies in which patients underwent staged neck dissection reported a $0 \%$ incidence of orocutaneous fistula. ${ }^{6,15}$ In addition, Holsinger et $\mathrm{al}^{17}$ reported zero cases of pharyngocutaneous fistula in 148 cases of patients undergoing conventional nonrobotic transoral lateral oropharyngectomy with concomitant neck dissection, suggesting that there may be a higher risk of intraoperative pharyngocervical communication with the use of the robot. Thus, although the rate of pharyngocutaneous fistula reported by Moore et $\mathrm{al}^{16}$ is objectively low and had relatively minor consequences, these data suggest that there may be a benefit to staged neck dissection. Of course, the potentially minor benefits of staged neck dissection must also be weighed against delayed management of neck disease, delayed planning for adjuvant therapy given the lack of pathologic data from neck dissection, delayed initiation of adjuvant therapy given the longer healing time needed, increased risk of a second general anesthetic, and increased costs of a second trip to the operating room. A randomized prospective study may better investigate these issues and determine the optimal management of the neck in patients undergoing TORS for oropharyngeal lesions.

Finally, studies comparing TORS to mandibulotomy for OPSCC unequivocally suggest that mandibulotomy carries a higher complication rate and a number of complications that simply do not occur with TORS. White et $\mathrm{al}^{8}$ compared rates of neck infection and pharyngocutaneous fistula following TORS and neck dissection ( $9 \%$ and $0 \%$, respectively) with those following open surgery ( $22 \%$ and $6 \%$, respectively) and found both rates to be lower with TORS, although the 
difference was only significant with neck infections, likely owing to the small absolute number of pharyngocutaneous fistulas. In addition, complications specific to mandibulotomy included free flap failure requiring operative intervention, mandibular malunion, bone exposure, and infections requiring hardware removal. ${ }^{5,8}$ Richmon et $\mathrm{ll}^{18}$ compared 116 TORS cases from 2008 to 2009 in the Nationwide Inpatient Sample with patients undergoing non-TORS surgical procedures for OPSCC and found that $0 \%$ of TORS patients and $44 \%$ of non-TORS patients had nonroutine hospital discharge, suggesting complications specific to open surgery.

\section{Positive margins}

The overall rate of positive margins on final pathology was found to be $1.5 \%-22 \% .^{5-10,12-15}$ Lee et al found no significant difference in margin positivity between TORS, conventional transoral surgery, and open surgery. Anecdotally, they did find that lesions in their series resected with TORS had greater inferior extension than those resected with conventional transoral surgery, and similar rates of margin positivity despite increased difficulty with transoral resection suggest a possible benefit of TORS. However, they also indicated that patients undergoing mandibulotomy had a significantly higher $\mathrm{T}$ stage on average. ${ }^{5}$ White et $\mathrm{al}^{8}$ found that in the salvage setting, there was a significantly lower rate of margin positivity with TORS than with open surgery (9\% vs 30\%, respectively), suggesting a potential benefit to TORS in this setting. No single study compared margin positivity across surgical techniques, while controlling for $\mathrm{T}$ stage, and those institutions performing both TORS and open surgery typically treated patients with higher T-stage lesions with mandibulotomy, but there was also no study suggesting that open surgery may have a lower rate of margin positivity, which is notable given that obtaining adequate access for negative margins has typically been viewed as the major challenge of transoral resection of oropharyngeal lesions.

\section{Airway management and tracheostomy dependence}

Rates of planned tracheostomy ranged from $0 \%$ to $59 \%$, and the majority of these patients were decannulated. $5,8-15,19,20$ Multiple recent large series reported that no routine tracheostomies were placed, and there were no instances of postoperative airway compromise. ${ }^{9,20}$ Although the rate of tracheostomy dependence following routine tracheostomy placement during TORS was very low, those patients requiring unplanned tracheostomy placement postoperatively were more likely to be dependent in the long term. Weinstein et al ${ }^{15,21}$ did not perform routine tracheostomy but left $6 / 27$ and 27/47 patients, respectively, intubated postoperatively, and these patients were extubated an average of 2.7-2.9 days postoperatively. Despite this strategy, they did report two unplanned postoperative tracheostomies for obstructive sleep apnea exacerbation and oropharyngeal edema, the latter of which remained tracheostomy-dependent. ${ }^{15}$ In addition, van Loon et $\mathrm{al}^{6}$ reported an unplanned tracheostomy for airway protection in the setting of postoperative major arterial hemorrhage requiring operative intervention, while Moore et $\mathrm{al}^{14}$ reported one patient that required tracheostomy placement during adjuvant therapy and remained tracheostomy-dependent. Studies comparing tracheostomy use with TORS with that of open surgery found that there were both a decreased need for tracheostomy placement ${ }^{8,18}$ and a shorter time to decannulation when tracheostomy was performed. ${ }^{5}$

\section{Swallowing and gastrostomy tube dependence}

Swallowing following treatment for OPSCC was found to be affected significantly by the treatment modalities used. Lee et $\mathrm{al}^{5}$ found that there was a significantly shorter time to swallow following TORS, as compared with mandibulotomy ( 7 days and 17 days, respectively). White et $\mathrm{al}^{8}$ similarly found a significantly decreased need for gastrostomy tube (GT) placement and shorter duration of use with TORS, even in the salvage setting. Richmon et $\mathrm{al}^{18}$ found that in the Nationwide Inpatient Sample, $0 \%$ of TORS patients and $19 \%$ of non-TORS patients required GT placement during management of their OPSCC. Studies reporting outcomes of definitive chemoradiation therapy describe comparable GT placement rates of $43 \%$ but higher long-term GT dependence in $5 \%-10 \%$ of patients. ${ }^{1,22}$

Although prophylactic GT (percutaneous or open) placement is no longer routine, many patients require temporary GT placement during adjuvant therapy. Weinstein et $\mathrm{al}^{21}$ reported prophylactic percutaneous endoscopic gastrostomy placement in all patients, 26/27 of whom were swallowing independently at last follow-up. Subsequent to this study, in candidates undergoing TORS as part of the primary therapy for their oropharyngeal cancer, GTs were placed either routinely or as a result of postoperative dysphagia in only $0 \%-6 \%$ of patients. ${ }^{5,7,12-14,20}$ On the other hand, up to $59 \%$ of patients undergoing adjuvant therapy required placement of a GT during radiation therapy; ${ }^{9-12,14,19}$ the majority of these were removed within the first year of treatment.

Al-Khudari et al found that the need for GT placement and long-term GT dependence both depended on disease 
stage, including salvage status, and the need for adjuvant therapy. In a series of 22 patients, they found that GTs were needed in $0 \%$ of patients with primary early (T1 or T2) disease, $40 \%-44 \%$ of patients with primary early or advanced (T3 or T4) disease undergoing adjuvant therapy, and $57 \%$ of patients undergoing salvage treatment. ${ }^{11}$ They also found that of those patients requiring GT placement, $50 \%$ of patients with primary early disease, $100 \%$ of patients with primary advanced disease, and 50\% of salvage patients were dependent in the long term. ${ }^{11}$ Dziegielewski et al found a number of risk factors associated with increased likelihood of the need for GT, including approximately fivefold increases in GT rates in patients with age $>55$ (potentially suggestive of lower baseline swallowing function) and resections involving more than one oropharyngeal subsite and 27-fold increases in long-term GT dependence in patients with pathologically T3-T4 tumors. ${ }^{12}$

Subjective swallow function following TORS has been assessed using a number of different questionnaires. Lee et al used the MDADI to compare swallow function at 12 months after TORS with conventional open surgery and found that patients undergoing TORS fared significantly better (76.1 and 64.9, respectively). ${ }^{5}$ Dziegielewski et al ${ }^{12}$ used the Head and Neck Cancer Inventory (HNCI) to determine health-related quality of life (HRQOL) in patients who underwent TORS. They found that eating function and eating attitude declined significantly after TORS (from 86.3 to 58.1 and 85.5 to 50.3 , respectively), with a nadir at 3 months and minimal recovery by 12 months. Leonhardt et al used the performance status scale (PSS) and found that there was a similarly significant decline in the eating and diet domains at 6 months but with partial recovery of reported function by 12 months. They observed a trend toward many scores being better with surgery alone than with surgery and postoperative RT, and still worse with the addition of chemotherapy. There were statistically significant differences in the PSS diet and eating score at 6 months between TORS alone (95.6 and 88.9, respectively) and TORS and chemoradiation (42.0 and 55.0, respectively). Other comparisons did not reach statistical significance likely owing to small sample size. ${ }^{19}$ Genden et $\mathrm{al}^{22}$ compared outcomes of TORS in 30 patients with those of definitive chemoradiation therapy in 26 patients, the majority of whom had oropharyngeal lesions. They demonstrated significantly better PSS eating and diet outcomes and Functional Oral Intake Scores at 2 weeks post-treatment with TORS. There were no significant differences at 3 months, 6 months, 9 months, or 12 months, but patients receiving chemoradiation remained below baseline even at 12 months.
Thus, swallowing after TORS is certainly affected for at least 2 weeks after surgery, and there is some evidence to suggest that subsequent outcomes may be improved if the patient is spared adjuvant therapy and in comparison with definitive chemoradiation therapy.

\section{Speech outcomes}

TORS has typically been thought to have minimal impact on speech. Genden et $\mathrm{al}^{22}$ found no significant change from baseline in the PSS Speech domain following TORS or chemoradiation therapy and no significant difference between the two groups. Similarly, Lee et $\mathrm{al}^{5}$ found no significant difference in reported voice outcomes at 12 months using the MDADI between patients undergoing TORS or mandibulotomy. On the other hand, Leonhardt et al reported a significant decline in the PSS speech domain at 6 months (99.3-86.2), with minimal recovery by 12 months, but there was no decline in patients treated with TORS alone. ${ }^{19}$ Dziegielewski et al ${ }^{12}$ found a similar decrease in the HNCI-HRQOL speech function and speech attitude domains, with moderate improvement between 6 months and 12 months, but they reported that based on established qualitative classifications of degree of clinically important differences (CID) for the HNCI domains that the CID was small. The minimal impact of TORS on speech is thought to be secondary to frequent sparing of the base of tongue and soft palate, regions that function in articulation; ${ }^{12}$ however, these regions were involved in at least one-third of cases in the series reported by Genden et $\mathrm{al}^{22}$ and Dziegielewski et al, ${ }^{12}$ so further investigation into the mechanisms of articulation is needed to be able to better predict which TORS patients are likely to be most affected.

\section{Overall health}

Two studies reported on overall health outcomes in patients undergoing TORS. Leonhardt et al administered the Short Form-8 Health Survey and found no significant changes after TORS in the physical functioning, role physical, mental health, and summary mental components over the follow-up duration. There was a decline in the bodily pain score at 6 months that recovered by 1 year. ${ }^{19}$ On the other hand, Dziegielewski et al ${ }^{12}$ found that the HNCI-HRQOL overall function, overall attitude, aesthetic attitude, and social function all dropped significantly by 3 months and recovered only partially, with overall function and overall attitude remaining significantly lower at 12 months. Thus, further investigation is needed to better assess the impact of TORS on perceived overall health, particularly in comparison with definitive chemoradiation or open surgery. 


\section{Adjuvant therapy and oncologic outcomes}

A number of studies compared oncologic outcomes of TORS with open surgery. Although Lee et al reported no significant difference in 2-year overall survival (OS) and disease-free survival (DFS), ${ }^{5}$ multiple other studies demonstrated a dramatic difference. ${ }^{8,23}$ Ford et al compared 65 patients with OPSCC treated with TORS with 65 patients who underwent open surgery and similar adjuvant therapy and found that DFS at 1 year, 2 years, and 3 years was 94\%, 91\% and 89\%, respectively, with TORS, and $85 \%, 75 \%$, and $73 \%$ with open surgery. ${ }^{23}$ White et $\mathrm{al}^{8}$ found an even larger difference in 2 -year DFS in the salvage setting (73\% with TORS, as compared with $43 \%$ with open surgery). One factor in improved DFS with TORS may be improved surgery-to-RT and total treatment package times. Carpenter et al reported a median of 41 days from TORS to the start of adjuvant therapy and 86 days until completion. Prior studies reported median surgery-to-RT times of 47-63 days following conventional surgery for HNSCC and total treatment package times of 94-108 days. ${ }^{24}$

There has been no randomized controlled trial comparing outcomes of TORS with definitive chemoradiation therapy. However, data from published case series all suggest that there is no difference in outcomes. Genden et al ${ }^{22}$ reported rates of 18-month locoregional control (LRC), distant control, DFS, and OS of $91 \%, 93 \%, 78 \%$, and $90 \%$, respectively, following TORS and $94 \%, 92 \%, 88 \%$, and $100 \%$, respectively, following IMRT in their series, with no significant difference between the groups. De Almeida et al conducted a systematic review of mostly single-institution studies reporting on IMRT for early T-stage OPSCC and reported 2-year DFS and OS of $82 \%-90 \%$ and $84 \%-96 \%$, respectively. ${ }^{1}$ These rates correspond to our findings of 2-year DFS and OS of 79\%-96\% and $82 \%-100 \%$, respectively, (Table 2 ) in patients undergoing TORS for previously untreated OPSCC.

A number of studies reported on outcomes following TORS with deintensified adjuvant therapy or without adjuvant therapy in patients with favorable pathologic features. Weinstein et $\mathrm{al}^{25}$ investigated the potential for deintensified postoperative radiation therapy in a subset analysis of the University of Pennsylvania Transoral Robotic Surgery Trial and found that in patients undergoing TORS with selective neck dissection who were not found to have any adverse pathologic features, deintensified adjuvant therapy was a viable treatment strategy, with one regional recurrence and one distant recurrence in 31 patients. Choby et al reported on 34 patients and found a 14-month DFS of $94 \%$ and
OS of $94 \% .{ }^{20}$ Weinstein et $\mathrm{a}^{13}$ reported on 30 patients and found a 18 -month LRC rate of $87 \%$ and OS of $100 \% .{ }^{13}$ It is notable that in the first study, the two recurrences and one death from disease were all in patients who had been recommended adjuvant therapy and refused; in the latter, two of four recurrences were in such patients. ${ }^{13,20}$ Olsen et al demonstrated similar results, with 2-year DFS of 78\%. ${ }^{26}$ These results suggest TORS alone without adjuvant therapy may be a viable treatment strategy for a subset of patients with favorable pathologic features.

\section{Human papillomavirus and smoking status}

A number of studies have reported on the impact of human papillomavirus (HPV) positivity and smoking status on TORS outcomes. In a small cohort, Cohen et $\mathrm{al}^{27}$ reported no significant difference in 2-year disease-specific survival (DSS) by HPV status. Ford et $\mathrm{al}^{23}$ compared outcomes of TORS with open surgery, stratified by HPV status, and found that while there was no difference in outcomes in patients who are HPV positive, patients who are HPV negative may benefit from TORS, with 1-year, 2-year, and 3-year DFS falling to $58 \%, 25 \%$, and $25 \%$, respectively, with open surgery. Finally, Stucken et al ${ }^{28}$ studied the effects of both HPV and smoking status. They demonstrated no significant difference in outcomes by smoking status alone, but HPV positivity was associated with significantly better 2-year LRC and DFS (100\% and $95 \%$, respectively), as compared with patients who are HPV negative ( $87 \%$ and $74 \%$, respectively). This relationship was further stratified by smoking status, where LRC and DFS in smokers in each group were worse than that of nonsmokers.

\section{Larynx}

TORS is also being used to manage patients with laryngeal carcinomas. The specific indications include supraglottic cancers and some glottis cancers that are amenable to a transoral resection. Cancers involving both arytenoids are not considered candidates for this surgery. Additionally, patients with poor pulmonary reserve are not candidates as aspiration is a significant postoperative risk.

\section{Patient sample}

A total of nine papers were reviewed regarding laryngeal surgery with the surgical robot. ${ }^{29-37}$ The total sample included 116 patients who had either a glottic or a supraglottic squamous cell carcinoma. Articles were included from the larger literature review (67 articles) if the purpose of the article was specific to the larynx subsite. Sample sizes for 
the studies ranged from 3 to 18 patients, all of which were reviewed retrospectively. Average follow-up ranged from 6.8 months to 28.1 months. Table 3 summarizes the articles reviewed and the perioperative, functional, and oncologic outcomes. The majority of studies evaluated T1 and T2 carcinomas with three studies also including select $\mathrm{T} 3$ cancers. Two articles evaluated glottic carcinomas.

\section{Perioperative outcomes}

Among the studies reviewing the supraglottic carcinoma population, neck dissections were performed for patients with node positive disease. Mendelsohn et al performed a sentinel lymph node biopsy in the node negative patient and a staged neck dissection if the sentinel node was positive. ${ }^{29}$ Olsen et al ${ }^{13}$ and Kayhan et al ${ }^{31}$ performed neck dissections in all patients. No immediate perioperative complications were noted among the studies, although three study populations did have patients requiring a tracheotomy postoperatively due to airway edema. ${ }^{31,34,35}$ One study noted five $(27.8 \%)$ perioperative complications without further specifics. ${ }^{29}$ Other delayed complications included a delayed tracheotomy bleed, supraglottic stenosis, ${ }^{33}$ and laryngeal stenosis. ${ }^{31}$ When reported, the average LOS ranged between 3.9 days and 18.6 days.

Two studies reviewed glottic carcinomas. The patients all presented with early stage T1-T2 tumors, and only one patient underwent a neck dissection with evidence of clinically positive nodal disease. Among this population, complications included postoperative need for tracheotomy in both groups. Otherwise, the LOS was shorter than the supraglottic group with an average stay between 4.1 days and 5 days. . $^{35,36}$

Two studies compared different surgical modalities to TORS. Park et al ${ }^{38}$ compared conventional open supraglottic partial laryngectomy to TORS and found that the TORS group had a significantly shorter hospital stay and return to oral diet and quicker decannulation as compared to conventional open supraglottic laryngectomy. Ansarin et al ${ }^{37}$ demonstrated similar results between TORS and transoral laser surgery with decreased operative time among the TORS group; however, the authors noted increased difficulty with visualizing the tumor in the TORS group.

\section{Functional outcomes}

In the case series by Mendelsohn et al, all 18 patients were kept intubated overnight after the surgery, and all were successfully extubated on postoperative day 1 . No patient required a tracheostomy, and no patient required a GT, although patients did have a nasogastric tube placed at the

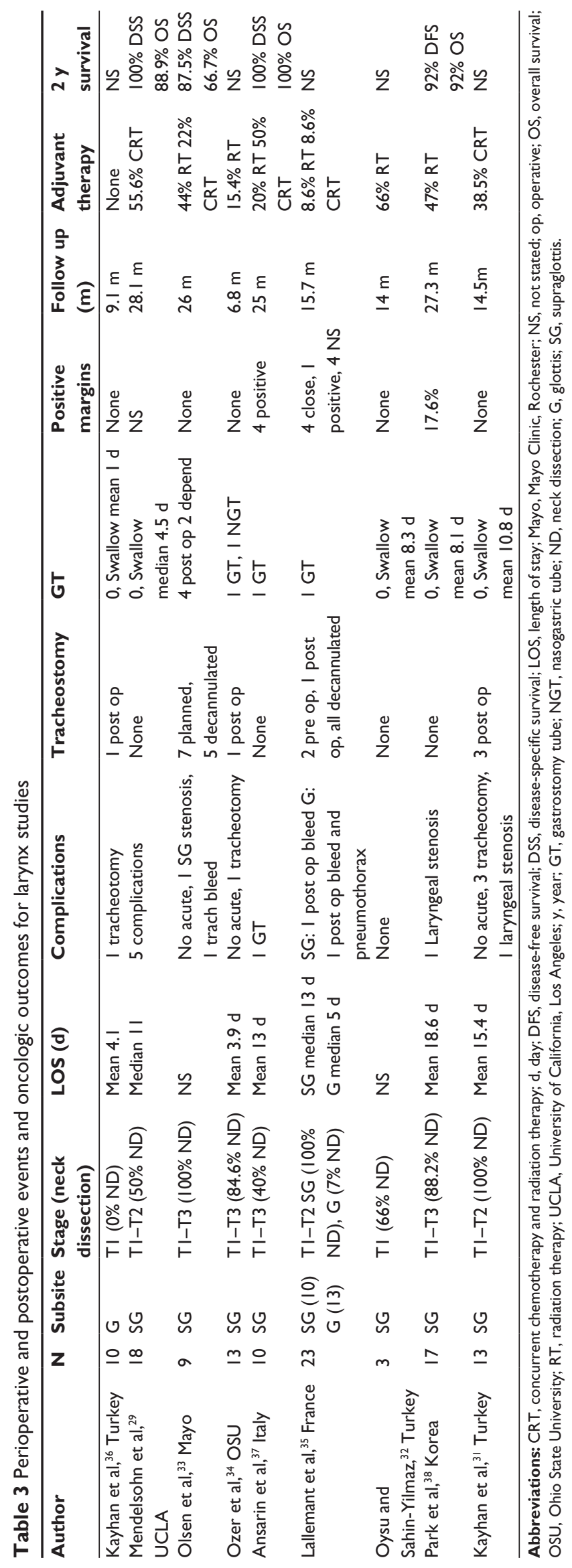


time of the operation. Patients ranged between 2 days and 29 days before demonstrating a safe swallow with solids and between 2 days and 45 days for thin liquids. ${ }^{29}$ In the patient population described by Olsen et al, all patients had a nasogastric tube placed intraoperatively, and seven had a planned tracheotomy. Five were decannulated after a 48-hour capping trial, and four patients required eventual GT placement, with two demonstrating long-term GT dependence. ${ }^{33}$ The other studies looking at supraglottic resections demonstrated patients tolerating an oral diet on average between 5 days and 10 days after surgery. ${ }^{29-32}$ The majority of patients were not GT-dependent and were swallowing within 2 weeks.

\section{Oncologic outcomes}

Among the patients by Mendelsohn et al, ten patients had nodal disease, all of whom underwent adjuvant chemoradiation for disease greater than N2a. Five of these patients demonstrated nodal extracapsular extension. The average follow-up for the group was 28.1 months with zero patients demonstrating local recurrence, three with regional recurrence, and four with distant recurrence. The 2-year DSS was $100 \%$, and OS was $88.9 \%$ with two patients dying of cardiopulmonary failure. ${ }^{29}$ In the series by Olsen et al, none of the patients demonstrated positive margins on resection, although four patients required intraoperative re-resection to clear the margins. A total of $66 \%$ of patients required adjuvant therapy, with $22 \%$ receiving chemoradiation. ${ }^{33}$ Ozer et al also demonstrated no positive margins on pathology, with two patients receiving adjuvant therapy for N2b disease. No patient had active disease at the end of follow-up, although the mean follow-up was only 6.8 months. ${ }^{34}$ Ansarin et al demonstrated no recurrences during the follow-up in the TORS group, with four patients demonstrating positive margins that were treated with either resection or adjuvant therapy. A total of $70 \%$ of patients received adjuvant therapy with $20 \%$ receiving radiation alone. ${ }^{37}$ Park et al found $47 \%$ of patients received adjuvant therapy and the 2-year OS and DFS were $92 \% .^{30}$

While the reviewed literature demonstrates high survival rates for the follow-up period of each retrospective study, no study establishes survival rates beyond 2 years. Additionally, the sample size of each individual study is relatively small and largely looks at early stage (T1 and T2) tumors.

\section{The unknown primary}

In addition to the literature that is currently available regarding TORS for the management of oropharyngeal carcinomas, there is also a significant amount of literature devoted to expanding the indications and uses of surgical robots for other oncologic surgeries. However, the data regarding outcomes is relatively limited. In addition to management of oropharyngeal carcinomas, TORS is also being utilized in identifying and treating patients with an unknown primary of the head and neck region. These patients present with a neck mass showing evidence of squamous cell carcinoma on fine needle aspiration or biopsy without evidence of a clinically evident primary cancer. In addition to imaging (including CT, MRI, and/or PET), the patient undergoes an exam under anesthesia with directed biopsies that can include an ipsilateral or bilateral tonsillectomy. Some institutions have employed TORS to remove the primary if identified during this examination or perform a lingual tonsillectomy with the goal of identifying a subcentimeter carcinoma in the base of tongue.

Durmus et al evaluated 22 patients from a single institution with evidence of an unknown primary. In these patients, $40.9 \%$ had an identifiable primary based on PET/CT with a false positive rate of $18.2 \%$. Imaging with directed biopsies revealed the primary tumor site in a total of $54.5 \%$ of patients. ${ }^{39}$ With the addition of TORS lingual and palatine tonsillectomy, $77.2 \%$ of patients had a diagnosed primary tumor. All patients in this group underwent unilateral or bilateral neck dissections during the same operative procedure. All patients received adjuvant RT and $40.9 \%$ received chemotherapy as well. ${ }^{39}$ In a similar cohort of 47 patients (multiinstitution) evaluated by Patel et al, $38.3 \%$ of patients did not have an identifiable primary on prior physical exam, imaging, or biopsies. Of these patients, lingual tonsillectomy with or without palatine tonsillectomy with TORS was able to identify a primary in $72.2 \%$ of patients, and including all patients, TORS identified a primary in 34 patients $(72.3 \%) .{ }^{40}$

\section{Discussion}

Through this review, we attempt to comprehensively evaluate the functional and oncologic outcomes of TORS reported in the literature in the treatment of HNSCC broken down by subsite. Although useful in summarizing the current state of outcomes of TORS, this methodology inherently has a number of limitations. Most studies included were retrospective in nature and either lacked controls or lacked randomization, thus yielding a more descriptive than comparative perspective on TORS outcomes and making it difficult to draw conclusions about optimal patient management. In addition, individual institutions reported relatively small series, and variability in both clinical practice and reporting of outcomes across institutions made it difficult to perform a 
true meta-analysis. Finally, a handful of large institutions each published a number of case series with undoubtedly overlapping patient populations, and while an attempt was made to exclude studies reporting on duplicate patients, it was impossible to determine whether this was definitively the case.

\section{Conclusion}

Despite these limitations, we can safely conclude that TORS represents a now well-established modality for treating cancers of the upper aerodigestive tract. Its utility is especially evident for earlier stage cancers of the oropharynx by allowing complete surgical resection with limited need for free flap reconstruction and decreased LOS in the hospital. There is evidence to show that the oncologic resection is at least comparable with open surgery with decreased morbidity and some data to suggest the same or better quality of life outcomes with a lower complication profile as compared with open surgery. Some institutions are also demonstrating a reduced need for adjuvant therapies such as chemotherapy or radiation, though in a retrospective fashion.

Further experience is needed to determine the role of TORS in the treatment of hypopharyngeal and laryngeal cancers, subsites where a goal of organ preservation has directed treatment options toward nonsurgical regimens. With the nontrivial toxicity of RT administered to these regions, even with the more widespread use of intensitymodulated RT, TORS could offer a surgical alternative while preserving laryngeal function. Continued evaluation of TORS outcomes, particularly with prospective and comparative studies, is needed to determine its optimal role in the management of HNSCC.

\section{Disclosure}

The authors report no conflicts of interest in this work.

\section{References}

1. de Almeida JR, Byrd JK, Wu R, et al. A systematic review of transoral robotic surgery and radiotherapy for early oropharynx cancer: a systematic review. Laryngoscope. 2014;124(9):2096-2102.

2. US Food and Drug Administration. Available from: http://www.fda.gov. Accessed January 6, 2015.

3. ClinicalTrials.gov. Available from: http://www.clinicaltrials.gov. Accessed June 1, 2015.

4. Nichols AC, Yoo J, Hammond JA, et al. Early-stage squamous cell carcinoma of the oropharynx: radiotherapy vs. trans-oral robotic surgery (ORATOR) - study protocol for a randomized phase II trial. $B M C$ Cancer. 2013;13(1):133.

5. Lee SY, Park YM, Byeon HK, Choi EC, Kim S-H. Comparison of oncologic and functional outcomes after transoral robotic lateral oropharyngectomy versus conventional surgery for $\mathrm{T} 1$ to $\mathrm{T} 3$ tonsillar cancer. Head Neck. 2014;36(8):1138-1145.
6. Van Loon JWL, Smeele LE, Hilgers FJM, van den Brekel MWM. Outcome of transoral robotic surgery for stage I-II oropharyngeal cancer. Eur Arch Otorhinolaryngol. 2014;272(1):175-183.

7. Dabas S, Dewan A, Ranjan R, et al. Transoral robotic surgery in management of oropharyngeal cancers: a preliminary experience at a tertiary cancer centre in India. Int J Clin Oncol. 2015;20(4):693-700.

8. White H, Ford S, Bush B, et al. Salvage surgery for recurrent cancers of the oropharynx. JAMA Otolaryngol Head Neck Surg. 2013; 139(8):773.

9. Sinclair CF, McColloch NL, Carroll WR, Rosenthal EL, Desmond RA, Magnuson JS. Patient-perceived and objective functional outcomes following transoral robotic surgery for early oropharyngeal carcinoma. Arch Otolaryngol Head Neck Surg. 2011;137(11):1112-1116.

10. Genden EM, Park R, Smith C, Kotz T. The role of reconstruction for transoral robotic pharyngectomy and concomitant neck dissection. Arch Otolaryngol Head Neck Surg. 2011;137(2):151-156.

11. Al-Khudari S, Bendix S, Lindholm J, Simmerman E, Hall F, Ghanem T. Gastrostomy tube use after transoral robotic surgery for oropharyngeal cancer. ISRN Otolaryngol. 2013;2013:190364.

12. Dziegielewski PT, Teknos TN, Durmus K, et al. Transoral robotic surgery for oropharyngeal cancer: long-term quality of life and functional outcomes. JAMA Otolaryngol Head Neck Surg. 2013;139(11): 1099-1108.

13. Weinstein GS, Quon H, Newman HJ, et al. Transoral robotic surgery alone for oropharyngeal cancer. Arch Otolaryngol Head Neck Surg. 2012;138(7):628-634.

14. Moore EJ, Olsen SM, Laborde RR, et al. Long-term functional and oncologic results of transoral robotic surgery for oropharyngeal squamous cell carcinoma. Mayo Clin Proc. 2012;87(3):219-225.

15. Weinstein GS, O'Malley BW, Cohen MA, Quon H. Transoral robotic surgery for advanced oropharyngeal carcinoma. Arch Otolaryngol Head Neck Surg. 2010;136(11):1079-1085.

16. Moore EJ, Olsen KD, Martin EJ. Concurrent neck dissection and transoral robotic surgery. Laryngoscope. 2011;121(3):541-544.

17. Holsinger FC, McWhorter AJ, Menard M, Garcia D, Laccourreye O. Transoral lateral oropharyngectomy for squamous cell carcinoma of the tonsillar region. Arch Otolaryngol Head Neck Surg. 2005;131: 583-591.

18. Richmon JD, Quon H, Gourin CG. The effect of transoral robotic surgery on short-term outcomes and cost of care after oropharyngeal cancer surgery. Laryngoscope. 2014;124(1):165-171.

19. Leonhardt FD, Quon H, Abrahao M, O’Malley BW, Weinstein GS. Transoral robotic surgery for oropharyngeal carcinoma and its impact on patient-reported quality of life and function. Head Neck. 2012; 34(2):146-154.

20. Choby GW, Kim J, Ling DC, et al. Transoral robotic surgery alone for oropharyngeal cancer: quality-of-life outcomes. Arch Otolaryngol Head Neck Surg. 2015;141(6):499-504.

21. Weinstein GS, O’Malley BW Jr, Snyder W, Sherman E, Quon H. Transoral robotic surgery. Arch Otolaryngol Head Neck Surg. 2007; 133(12):1220-1226.

22. Genden EM, Kotz T, Tong CC, et al. Transoral robotic resection and reconstruction for head and neck cancer. Laryngoscope. 2011;121(8): $1668-1674$.

23. Ford SE, Brandwein-Gensler M, Carroll WR, Rosenthal EL, Magnuson JS. Transoral robotic versus open surgical approaches to oropharyngeal squamous cell carcinoma by human papillomavirus status. Otolaryngol Head Neck Surg. 2014;151(4):606-611.

24. Carpenter TJ, Kann B, Buckstein MH, et al. Tolerability, toxicity, and temporal implications of transoral robotic surgery (TORS) on adjuvant radiation therapy in carcinoma of the head and neck. Ann Otol Rhinol Laryngol. 2014;123(11):791-797.

25. Weinstein GS, Quon H, O'Malley BW, Kim GG, Cohen MA. Selective neck dissection and deintensified postoperative radiation and chemotherapy for oropharyngeal cancer: a subset analysis of the University of Pennsylvania transoral robotic surgery trial. Laryngoscope. 2010; 120(9):1749-1755. 
26. Olsen SM, Moore EJ, Laborde RR, et al. Transoral surgery alone for human-papillomavirus-associated oropharyngeal squamous cell carcinoma. Ear Nose Throat J. 2013;92(2):76-83.

27. Cohen M, Weinstein GS, O’Malley BW, Feldman M, Quon H. Transoral robotic surgery and human papillomavirus status: oncologic results. Head Neck. 2011;33(4):573-580.

28. Stucken CL, de Almeida JR, Sikora AG, Tong CCL, Genden EM The impact of human papillomavirus and smoking on survival outcomes after transoral robotic surgery. Head Neck. 2014. doi:10.1002/ hed.23915.

29. Mendelsohn AH, Remacle M, Van Der Vorst S, Bachy V, Lawson G. Outcomes following transoral robotic surgery: supraglottic laryngectomy. Laryngoscope. 2012;123(1):208-214.

30. Park YM, Byeon HK, Chung Pil H, Choi Chang E, Kim S-H. Comparison of treatment outcomes after transoral robotic surgery and supraglottic partial laryngectomy : our experience with seventeen and seventeen patients respectively. Clin Otolaryngol. 2013;38(3): 270-274.

31. Kayhan FT, Kaya KH, Altintas A, Sayin I. Transoral robotic supraglottic partial laryngectomy. J Craniofac Surg. 2014;25(4):1422-1426.

32. Oysu C, Sahin-Yilmaz A. En bloc resection of epiglottic tumors with transoral robotic approach - preliminary results. Int JMed Robot. 2013;9: 477-479.

33. Olsen SM, Moore EJ, Koch CA, Price DL, Kasperbauer JL, Olsen KD. Transoral robotic surgery for supraglottic squamous cell carcinoma. Am J Otolaryngol. 2012;33(4):379-384.
34. Ozer E, Alvarez B, Kakarala K, Durmus K, Teknos T, Carrau R. Clinical outcomes of transoral robotic supraglottic laryngectomy. Head Neck. 2013;35(8):1158-1161.

35. Lallemant B, Chambon G, Garrel R, et al. Transoral robotic surgery for the treatment of T1-T2 carcinoma of the larynx: preliminary study. Laryngoscope. 2013;123(10):2485-2490.

36. Kayhan FT, Kaya KH, Sayin I. Transoral robotic cordectomy for early glottic carcinoma. Ann Otol Rhinol Laryngol. 2012;121(8): 497-502.

37. Ansarin M, Zorzi S, Massaro MA, et al. Transoral robotic surgery vs transoral laser microsurgery for resection of supraglottic cancer: a pilot study. Int J Med Robot. 2014;10:107-112.

38. Park YM, Byeon HK, Chung Pil H, Choi Chang E, Kim S-H. Comparison of treatment outcomes after transoral robotic surgery and supraglottic partial laryngectomy: our experience with seventeen and seventeen patients respectively. Clin Otolaryngol. 2013;38(3): $270-274$.

39. Durmus K, Rangarajan S, Old M, Agrawal A, Teknos T, Ozer E. Transoral robotic approach to carcinoma of unknown primary. Head Neck. 2014;36(6):848-852.

40. Patel SA, Magnuson JS, Holsinger FC, et al. Robotic surgery for primary head and neck squamous cell carcinoma of unknown site. JAMA Otolaryngol Head Neck Surg. 2013;139(11):1203-1211.
Robotic Surgery: Research and Reviews

\section{Publish your work in this journal}

Robotic Surgery: Research and Reviews is an international, peer reviewed, open access, online journal publishing original research, commentaries, reports, and reviews on the theory, use and application of robotics in surgical interventions. Articles on the use of supervisory-controlled robotic systems, telesurgical devices, and shared-control systems are

\section{Dovepress}

invited. The manuscript management system is completely online and includes a very quick and fair peer review system, which is all easy to use. Visit http://www.dovepress.com/testimonials.php to read real quotes from published authors.

Submit your manuscript here: http://www.dovepress.com/robotic-surgery-research-and-reviews-journal 